\title{
Increasing the transverse mode coupling instability threshold by RF quadrupole
}

\author{
V. V. Danilov* \\ Fermi National Accelerator Laboratory, P.O. Box 500, Batavia, Illinois 60510
}

(Received 19 June 1998; published 17 August 1998)

\begin{abstract}
Transverse mode coupling instability is one of the major limitations of a single bunch current in storage rings. Until now it has appeared in large electron-positron machines, while its presence in proton colliders has not been observed. This paper describes a theoretical analysis of the effect of longitudinal variation of the betatron tune on the transverse mode coupling instability. This variation can be introduced by an RF quadrupole. In the result, the instability threshold could be significantly increased when a modulation of the betatron frequency is comparable with the synchrotron tune. [S1098-4402(98)00012-3]

PACS numbers: 29.27.Bd
\end{abstract}

\section{INTRODUCTION}

Recently [1] it was discovered by simulations that an incoherent tune shift can increase the threshold current for the fast head-tail instability. Probably one of the reasons for this is the dependence of the betatron tune on longitudinal coordinates. Earlier, a remarkable rise in the threshold current of transverse coupled-bunch instabilities was observed at the Photon Factory at KEK (Japan) using a high frequency quadrupole magnet [2]. An influence of betatron tune modulation on the transverse mode coupling instability (TMCI, see $[3,4]$ ) threshold is analyzed here.

For the purpose of this article, one transverse and one longitudinal degree of freedom is considered. The definitions of wake functions correspond to [4]; all of the results are obtained in general form. After that, a particular case with a constant wake function ${ }^{1}$ is studied.

The action of the RF quadrupole is expressed in dependence of the betatron frequency on the longitudinal coordinate

$$
\omega_{b}=\omega_{0}+s g,
$$

where $\omega_{0}$ is the initial betatron frequency, $s$ is the distance of a particle from the center of the bunch, and the longitudinal derivative of the betatron frequency $g$ is proportional to a strength of the RF quadrupole ${ }^{2}$.

It is shown below that the betatron frequency modulation comparable to the synchrotron tune increases the TMCI threshold by a factor of 3 . Then the threshold grows with the gradient $g$ growth.

The physical reason for such a phenomenon can be explained in the following way. During one-half of a

\footnotetext{
*On leave from Budker Institute of Nuclear Physics, Novosibirsk 630090, Russia.

${ }^{1}$ It corresponds, for example, to the wake function of a strip line.

${ }^{2}$ The wavelength of RF oscillations is assumed to be much larger than the bunch length.
}

synchrotron oscillation, head particles change the betatron phase of backward particles; when the change of the betatron phase over the synchrotron period is of the order of unity, this instability occurs. The betatron frequencies are usually the same for the head and the tail of a bunch, so the particles are always in resonance. When the longitudinal gradient of the betatron frequencies is applied, the particles get out of resonance, which suppresses the collective motion.

In the next section the simple model of a "hollow beam" is presented; then all of the calculations are carried out for a more practical Gaussian distribution.

\section{II. "HOLLOW-BEAM" MODEL}

It is usually convenient to study a simple model to understand general properties of eigenvalues and their dependence on parameters. At first we use a model of the bunch, which consists of particles with one synchrotron amplitude. The basic equation for a dipole moment motion in the presence of a distributed wakefield is

$$
D^{\prime \prime}+\omega_{b}^{2} D=F,
$$

where

$$
F=\frac{e^{2}}{L} \int_{s}^{\infty} W(\Delta s) D\left(s^{\prime}\right) \rho\left(s^{\prime}\right) d s^{\prime},
$$

$\Delta s$ is the distance between the forward and the test particle, $W$ is the wake function of a vacuum chamber, $D$ and $\rho$ are the average transverse dipole moment and density of the forward particles, and $L$ is the circumference of the machine.

More general wakefields can be considered with the help of averaged equations. Let $A$ and $\psi$ be the amplitude and slow phase of betatron oscillations of the test particle dipole moment for a fixed synchrotron phase. The averaged equation for them reads (the chromaticity and the longitudinal gradient of betatron frequencies is equal to zero)

$$
\frac{d A e^{i \psi}}{d \tau}=\frac{\omega_{b} \beta^{3 / 2}}{i \gamma m c^{2}} \int_{0}^{T} F e^{-i \omega_{b} \tau} \frac{d \tau}{T}=\bar{F},
$$


where the integration time $T$ must be larger than the betatron oscillation period; $\omega_{b}, \beta$ are the betatron frequency and the $\beta$ function, and $\omega_{b} d \tau=d z / \beta$, and the force $F$ is the same as in (3), but with arbitrary dependence of the wake function on time.

After averaging by using the relation $\omega_{b} d \tau=d z / \beta$, the equation for hollow beam (for zero longitudinal gradient of the betatron frequency) reads

$$
\frac{d A e^{i \psi}}{d \tau}=\frac{N e^{2} \beta}{i 4 \pi \gamma m c^{2} T_{0}} \int_{-|\phi|}^{|\phi|} W(\Delta s) \mathcal{D}\left(\phi^{\prime}\right) d \phi^{\prime},
$$

where the $\beta$ function is supposed to be constant for simplicity, $\phi$ is the synchrotron phase ${ }^{3}, N$ is the number of particles, $\Delta s=a \cos (\phi)-a \cos \left(\phi^{\prime}\right), T_{0}$ is the revolution frequency, and $\mathcal{D}=\frac{D}{\sqrt{\beta}}$ is the average normalized dipole moment $\mathcal{D}=A e^{i \psi}$.

For a nonzero longitudinal gradient of betatron frequencies, the slow betatron phase $\psi$ consists of two parts [see Eq. (1)]

$$
\psi=\Psi+g \int^{\tau} s d \tau=\Psi+\frac{g \lambda T_{0} E_{0}}{U} \delta,
$$

where $\tau$ is time, $\Psi$ is the slow part of the betatron phase, $\lambda$ and $U$ are the wavelength and amplitude of the RF system, $E_{0}$ is the energy of particles, $T_{0}$ is the revolution period, and $\delta=\Delta E / E_{0}$. It is convenient to use a new variable $A e^{i \Psi}$ because $A$ and $\Psi$ here are influenced only by a collective force. One can easily obtain the equation for this variable:

$$
\begin{aligned}
\frac{d A e^{i \Psi}}{d \tau}= & e^{-i \frac{g \lambda T_{0} E_{0}}{U} \delta} \frac{N e^{2} \beta}{i 4 \pi \gamma m c^{2} T_{0}} \\
& \times \int_{-|\phi|}^{|\phi|} W(\Delta s) \mathcal{D}\left(\phi^{\prime}\right) d \phi^{\prime} .
\end{aligned}
$$

Taking into account that $\mathcal{D}=A e^{i \Psi+i \frac{g \lambda T_{0} E_{0}}{U} \delta}$ and after rewriting the total derivative on time via partial derivatives on time and synchrotron phase, the previous equation converts to

$$
\begin{aligned}
\frac{\partial d}{\partial \tau}+\frac{\partial d}{\partial \phi}= & \frac{N e^{2} \beta}{i 4 \pi \gamma m c^{2} T_{0}} e^{-i P \sin (\phi)} \\
& \times \int_{-|\phi|}^{|\phi|} W(\Delta s) d\left(\phi^{\prime}\right) e^{i P \sin \left(\phi^{\prime}\right)} d \phi^{\prime},
\end{aligned}
$$

where $d=A e^{i \Psi}$ and parameter $P$ is half of the differences of betatron phases between maximum and minimum energy offsets for this particular synchrotron amplitude [the amplitude and the relative energy offset are related to each other by the formula $\delta=a_{e} \sin (\phi)$ ]

$$
P=\frac{g \lambda T_{0} E_{0}}{U} a_{e} .
$$

Then for finding eigenfrequencies it is convenient to present $d$ in the form of an infinite sum of harmonics

\footnotetext{
${ }^{3}$ The module $\phi$ in this formula was written due to the symmetry of the collective force on the synchrotron phase.
}

of synchrotron frequency multiplied by the exponent function of time

$$
d=e^{i \alpha \tau} \sum_{n=-\infty}^{+\infty} d_{n} e^{-i n \phi},
$$

where $\alpha$ is some eigenfrequency. After putting it in the previous equation, multiplying it by $e^{i n \phi}$, integrating the equation over the synchrotron phase from $-\pi$ to $\pi$, and rearranging the terms, one can obtain

$$
d_{n}\left(\alpha-n \omega_{s}\right)=-K \sum_{m=-\infty}^{+\infty} d_{m} K_{n m},
$$

where $\omega_{s}$ is the synchrotron frequency, $K=N e^{2} \beta /$ $2 \pi^{2} \gamma m c^{2} T_{0}$, and $K_{n m}=\int_{0}^{\pi} \cos [n \phi-P \sin (\phi)] d \phi \times$ $\int_{0}^{\phi} W(\Delta s) \cos \left[m \phi^{\prime}-P \sin \left(\phi^{\prime}\right)\right] d \phi^{\prime}$.

Usually the sum is truncated to a finite number of lower modes. In the case of only two lower modes $n=0,-1$, the matrix for finding eigenfrequencies is

$$
\left(\begin{array}{cc}
\alpha+K_{00} K & K_{01} K \\
K_{10} K & \alpha+\omega_{s}+K_{-1-1} K
\end{array}\right) .
$$

Setting the determinant of this matrix equal to zero, one can obtain an equation for $\alpha$. For zero $P, K_{01}=$ $-K_{10}$ and an imaginary part of eigenfrequencies appears for some threshold current (see, for example, [3]). It is evident that when $K_{01}, K_{10}$ have the same sign, this quadratic equation never gives imaginary solutions, so the TMCI instability in this model disappears. Figure 1 shows $K_{01}, K_{10}$ versus parameter $P(8)$ for constant wake $W=1$. For $P \simeq 0.77$, the coupling terms are of the same sign and TMCI disappears for such a simple model. For another sign of $P$, the figure of $K_{01}, K_{10}$ versus $P$ can be obtained by the reflection of Fig. 1 over the zero $x$ axis, so the threshold depends on the modulus $P^{4}$.

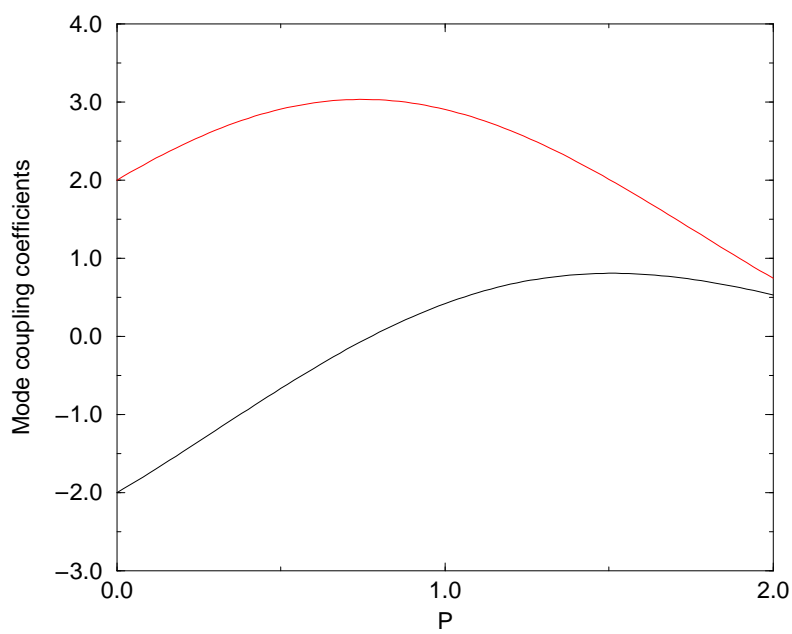

FIG. 1. (Color) Coefficients $K_{01}$ (upper) and $K_{10}$ (lower) versus the parameter $P$ [see Eq. (8)].

\footnotetext{
${ }^{4}$ The same is valid for all of the following results.
} 


\section{GENERAL INVESTIGATION}

The equation for Gaussian distribution in synchrotron phase space can be obtained in the same manner:

$$
\begin{aligned}
\frac{\partial d(a, \phi)}{\partial \tau}+\frac{\partial d(a, \phi)}{\partial \phi}= & \frac{N e^{2} \beta}{i 4 \pi \gamma m c^{2} T_{0} \sigma^{2}} e^{-i P_{\sigma} a / \sigma \sin (\phi)} \int_{0}^{\infty} a^{\prime} d a^{\prime} \exp \left[-\left(a^{\prime}\right)^{2} / 2 \sigma^{2}\right] \\
& \times \int_{-F\left(a, a^{\prime},|\phi|\right)}^{F\left(a, a^{\prime},|\phi|\right)} W(\Delta s) d\left(a^{\prime}, \phi^{\prime}\right) e^{i P_{\sigma} a^{\prime} / \sigma \sin \left(\phi^{\prime}\right)} d \phi^{\prime},
\end{aligned}
$$

where $a$ is the synchrotron amplitude, $\sigma$ is the longitudinal rms size, $\Delta s=a \cos (\phi)-a^{\prime} \cos \left(\phi^{\prime}\right)$, and $F\left(a, a^{\prime}, \phi\right)$ is determined by

$F\left(a, a^{\prime}, \phi\right)=a \cos \left[a / a^{\prime} \cos (\phi)\right], \quad$ if $\left|a / a^{\prime} \cos (\phi)\right|<1$;

$$
F\left(a, a^{\prime}, \phi\right)=\pi \operatorname{sgn}[\cos (\phi)], \quad \text { otherwise . }
$$

$P_{\sigma}$ is the half of the maximum betatron phase difference for particles with the positive and negative energy offsets for $a_{e}=\sigma$,

$$
P_{\sigma}=\frac{g \lambda T_{0} E_{0}}{U} \sigma
$$

For practical calculations of the eigenvalues of this problem, the bunch is divided into rings with the fixed amplitudes in the synchrotron phase space. So the dimension of this system increases in factor equal to the number of the rings in comparison with the hollowbeam model. The coupling coefficient of some mode with azimuthal number $n$ and amplitude $a$ with some mode with azimuthal number $m$ and amplitude $b$ is

$$
K_{n m a b}=R \int_{0}^{\pi} \cos \left[n \phi-P_{\sigma} \sin (\phi) a / \sigma\right] d \phi \int_{0}^{F(a, b, \phi)} W(\Delta s) \cos \left[m \phi^{\prime}-P_{\sigma} \sin \left(\phi^{\prime}\right) b / \sigma\right] d \phi^{\prime},
$$

where $R=N e^{2} \beta b \exp \left(-b^{2} / 2 \sigma^{2}\right) / 2 \pi^{2} \gamma m c^{2} T_{0} \sigma^{2}$.

The linear equation for eigenmodes is

$$
d_{n}\left(a_{i}\right)\left(\alpha-n \omega_{s}\right)=-\sum_{j} \sum_{m=-\infty}^{+\infty} d_{m}\left(a_{j}\right) K_{n m a_{i} a_{j}},
$$

where $\alpha$ is the eigenfrequency, and $i, j$ are the indices of the rings in synchrotron phase space. The smaller the rings are, the closer the eigenvalues are to their actual values. They can be found from the equation, which can be obtained after setting the determinant from the above matrix equal to zero.

Further, the results of the eigenvalue calculations are shown. The bunch is divided into five radial rings, and each ring is presented by five azimuthal modes, so it is

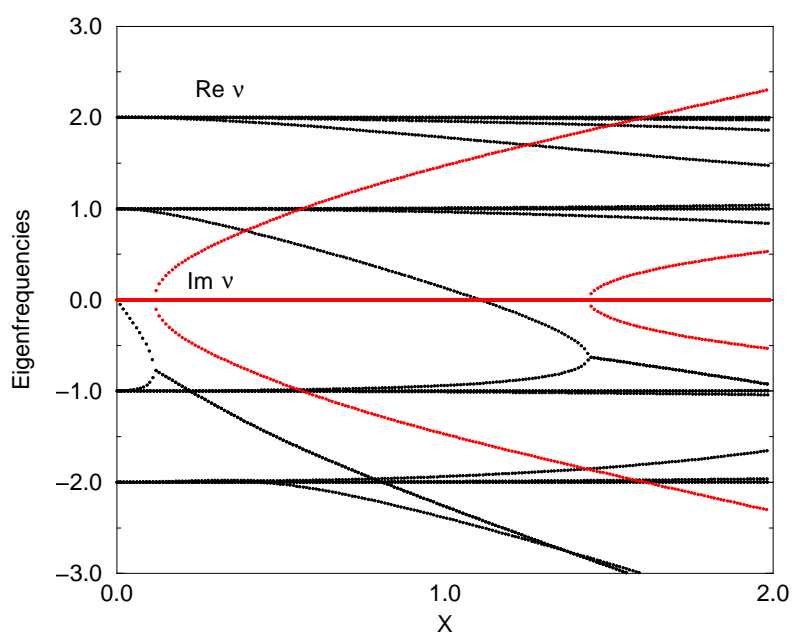

FIG. 2. (Color) Eigenfrequencies (real parts in black, imaginary in red) of transverse oscillations versus $X=$ $N e^{2} \beta W / 2 \pi^{2} \gamma m c^{2} T_{0}$. The parameter $P_{\sigma}=0$. possible to see the behavior of the first 25 modes, which usually gives a few percent deviation for the threshold from its actual value.

In Fig. 2, one can see the eigentunes for the constant wake function $W$ versus $X=N e^{2} \beta W / 2 \pi^{2} \gamma m c^{2} T_{0}$, which is proportional to the number of particles per bunch. In this figure, the betatron tune corresponds to zero at the vertical axis. All of the tunes are expressed in terms of the synchrotron tune, and the parameter $P_{\sigma}$ (11) is equal to zero. All of the real parts of frequencies start from a zero current; the imaginary parts appear near the zero axis after the merging of some modes. Near the betatron tune, there is a bunch of the "radial" eigentunes with a zero "azimuthal" number. If, briefly, all of these

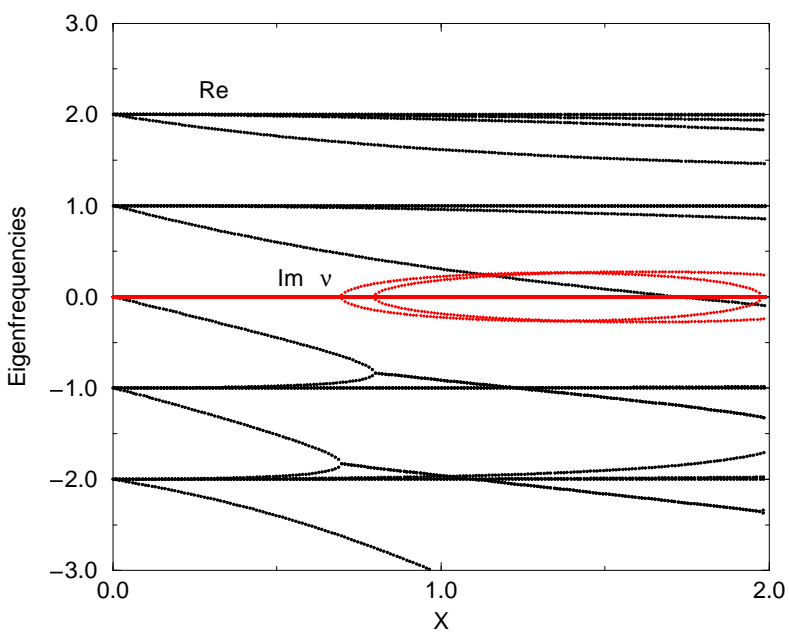

FIG. 3. (Color) Eigenfrequencies (real parts in black, imaginary in red) of transverse oscillations versus $X=$ $N e^{2} \beta W / 2 \pi^{2} \gamma m c^{2} T_{0}$. The parameter $P_{\sigma}=2$. 


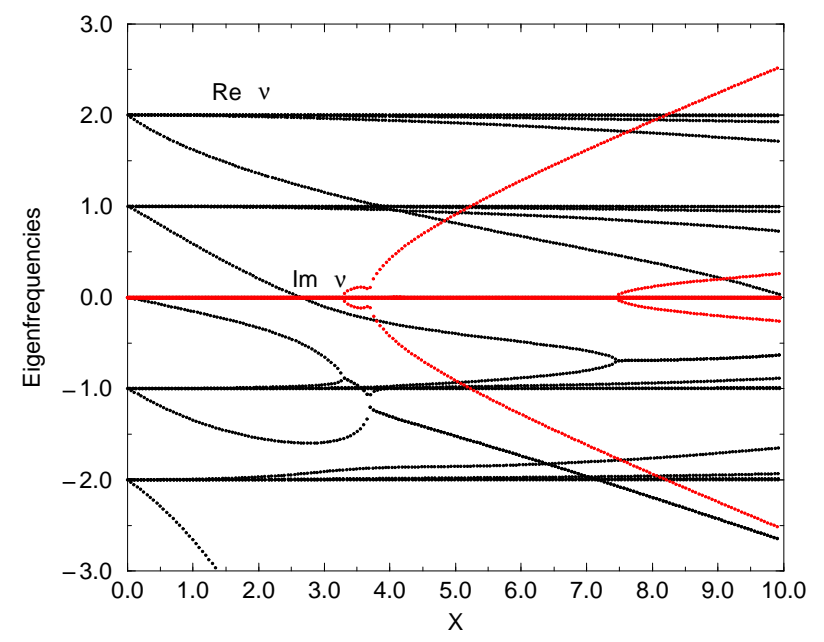

FIG. 4. (Color) Eigenfrequencies (real parts in black, imaginary in red) of transverse oscillations versus $X=$ $N e^{2} \beta W / 2 \pi^{2} \gamma m c^{2} T_{0}$. The parameter $P_{\sigma}=5$.

modes for a small current have zero oscillations of the dipole moment over an angle in the synchrotron phase space, and they differ in dependence of the dipole moment on the synchrotron amplitude (radius in synchrotron phase space). For each integer number, there are higher azimuthal modes whose tunes differ from the betatron tune in this particular integer number of synchrotron tunes for a small current. This number means the number of modulation of eigenmodes over the angle in synchrotron phase space. As in the case of "zero" azimuthal modes, there are a lot of radial modes for every azimuthal number. The first merging of a zero azimuthal mode and a " -1 " radial mode occurs for $X=0.12$. The next merging occurs for a five times larger current $(-1$ and +1 modes). In both cases, a pair of the modes with equal real parts of the tune and with opposite imaginary parts of the tune appears; this, evidently, means instability of the bunch.

Figures 3 and 4 show the eigenvalues for $P_{\sigma}=2$ and $P_{\sigma}=5$, consequently. Finally, the factor of increasing the TMCI threshold is shown in Fig. 5. One can see that the RF quad can raise easily the threshold in factor five and more.

\section{CONCLUSION}

The longitudinal gradient of the betatron frequency can effectively suppress the TMCI instability as it does for the transverse instabilities in linacs [6].

Rough estimations for the very large hadron collider (VLHC) [5] can be made for the length of such a quadrupole. Let us take the maximum electric field equal

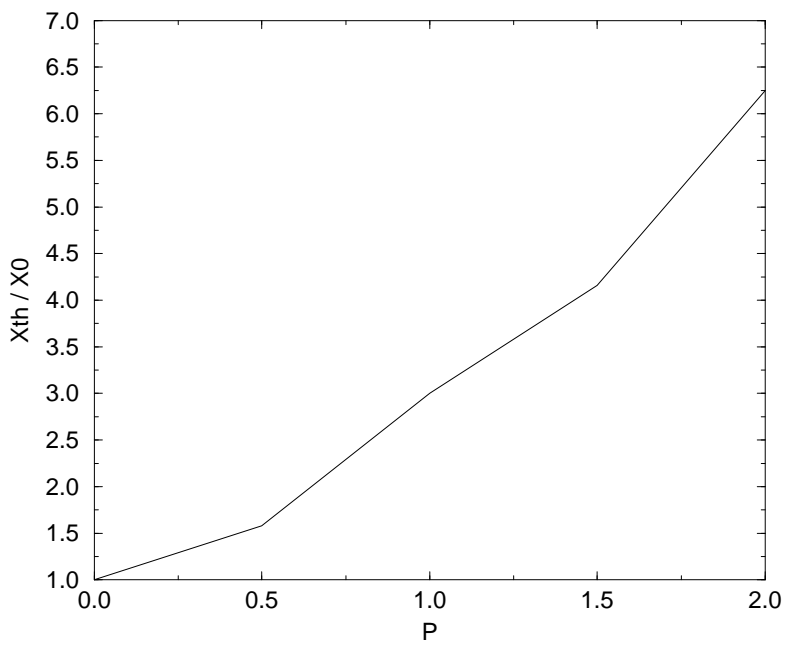

FIG. 5. The factor of increasing the TMCI threshold versus parameter $P$

to $5 \mathrm{MV} / \mathrm{m}$, aperture and wavelength of $\mathrm{RF}$ equal to $10 \mathrm{~cm}$ (the designed bunch length is approximately the same), $\beta$ function equal to $600 \mathrm{~m}$, the synchrotron tune equal to 0.01 , and the injection energy is $3 \mathrm{TeV}$. The $12.5 \mathrm{~m}$ quadrupole is needed to produce the betatron tune spread of about one synchrotron tune. It seems possible to combine RF quadrupole with the basic RF system. In this case, the same RF generators can be used. So the TMCI threshold can be suppressed by means of a conventional technique.

\section{ACKNOWLEDGMENTS}

The author thanks V. Ptitsin for pointing out the paper [1] and M. Blaskiewicz for valuable comments. The author also acknowledges useful discussions with V. Shiltsev and the help of A. Burov and B. Zotter in the preparation of the final version of this paper.

[1] M. Blaskiewicz, in 1997 Particle Accelerator Conference, Vancouver, Canada (to be published).

[2] S. Sakanaka, T. Mitsuhashi, A. Ueda, M. Izawa, Nucl. Instrum. Methods Phys. Res., Sect. A 325, 1 (1993).

[3] V. V. Danilov and E. A. Perevedentsev, Nucl. Instrum. Methods Phys. Res., Sect. A 391,77 (1997).

[4] A.W. Chao, Physics of collective beam instabilities in high energy accelerators (Wiley, New York, 1993).

[5] Proceedings of the 1996 DPF/DPB Summer Study on High-Energy Physics, Snowmass, Colorado (Stanford Linear Accelerator Center, Menlo Park, CA, 1997)

[6] V.E. Balakin, A. V. Novokhatsky, and V.P. Smirnov, Proceedings of the 12th International Conference On High-Energy Accelerators (Fermilab, Batavia, IL, 1983). 